variation in pollution levels compared with between-city variation.

Original article Miller KA et al. (2007) Long-term exposure to air pollution and incidence of cardiovascular events in women. N Engl J Med 356: 447-458

\section{A network meta-analysis of the effects of antihypertensive agents on incident diabetes}

Several meta-analyses have attempted to assess the effects of different classes of antihypertensive agents on incident diabetes, but this approach has been hampered by trial heterogeneity, and a lack of comparison between angiotensin-converting-enzyme (ACE) inhibitors and angiotensin-receptor blockers (ARBs). Elliott and Meyer report on a network meta-analysis of data from 22 randomized controlled trials. The network meta-analysis technique enabled indirect comparison of the effects of ACE inhibitors and ARBs on incident diabetes.

In comparison with initial treatment with a diuretic, initial treatments with $A R B s$ (odds ratio [OR] 0.62; $P<0.0001)$, ACE inhibitors (OR $0.67 ; P<0.0001$ ), placebo (OR $0.75 ; P=0.001$ ) or calcium-channel blockers (OR 0.79; $P=0.004$ ) were each associated with a significantly reduced risk of incident diabetes. The rate of incident diabetes after treatment with $\beta$-blockers was not significantly different from that seen after initial treatment with a diuretic (OR 0.93; $P=0.43$ ). These results were robust to multiple one-way sensitivity analyses. The degree of incoherence of the network was 0.054 , indicating that the model might have been internally consistent despite including data from heterogeneous studies.

Individual pairwise comparison of ARBs and ACE inhibitors did not detect a significant difference in incident diabetes rate $(P=0.16)$. Several trials with incident diabetes as a prespecified end point are in progress, and these might help to clarify the glycemic effects of antihypertensive agents - particularly those directly inhibiting the renin-angiotensin system-as well as the association of incident diabetes with subsequent cardiovascular risk.

Original article Elliott WJ and Meyer PM (2007) Incident diabetes in clinical trials of antihypertensive drugs:

a network meta-analysis. Lancet 369: 201-207; Erratum.

Lancet, in press

\section{Ultrafiltration - a highly effective treatment for hypervolemic heart failure}

Hypervolemia leads to disease progression and mortality in patients with heart failure. Intravenous diuretic therapy is the current standard of care for hypervolemic heart failure, but loop diuretics decrease in effectiveness with repeated use. In addition, several studies have linked these diuretics to increased morbidity and mortality owing to adverse effects on neurohormonal activation, electrolyte balance and cardiac and renal function. Now, in a prospective, multicenter trial, the UNLOAD (Ultrafiltration versus Intravenous Diuretics for Patients Hospitalized for Acute Decompensated Congestive Heart Failure) trial investigators have demonstrated the safety and efficiency of ultrafiltration in the treatment of decompensated heart failure.

In this study, 200 patients hospitalized for heart failure and with two or more signs of hypervolemia were randomized within $24 \mathrm{~h}$ of admission to ultrafiltration or intravenous diuretics. At $48 \mathrm{~h}$ after randomization, weight loss and net fluid loss were significantly higher in the ultrafiltration group than in the group receiving intravenous diuretics ( $P=0.001$ for both). At 90 days, fewer patients from the ultrafiltration group than from the intravenous diuretic group had been rehospitalized for heart failure (18\% vs $32 \%$; $P=0.037)$. Furthermore, fewer rehospitalization days and per patient unscheduled visits were recorded in the ultrafiltration group. Throughout the study, serum creatinine levels were similar in the two groups, and no clinically significant changes were seen in serum blood urea nitrogen, sodium, chloride or bicarbonate in either group.

The UNLOAD trial clearly demonstrates that ultrafiltration is an effective alternative to intravenous diuretic therapy in patients with hypervolemic heart failure.

Original article Costanzo MR et al. for the UNLOAD Trial Investigators (2007) Ultrafiltration versus intravenous diuretics for patients hospitalized for acute decompensated heart failure. J Am Coll Cardiol 49: 675-683

\section{Aspirin therapy is safe in cancer patients with ACS and thrombocytopenia}

The benefits of aspirin therapy in patients with acute coronary syndromes (ACS) have been 